\title{
Breakup of a supported drop of a viscous conducting liquid in a uniform electric field
}

\author{
F. J. Higuera \\ E. T. S. Ingenieros Aeronáuticos, UPM Plaza Cardenal Cisneros 3, 28040 Madrid, Spain
}

(Received 11 February 2008; published 23 July 2008)

\begin{abstract}
Numerical computations and order-of-magnitude estimates are used to describe the time evolution of a drop of a very viscous liquid of finite electrical conductivity attached to a metallic plate which is suddenly subject to a uniform electric field. Under the action of the electric stresses induced at its surface, the drop elongates in the direction of the field, and charged droplets are emitted when the strength of the field is higher than a certain critical value. A stationary emission mode exists in which the attached drop develops a conical tip and a thin jet, with small droplets emitted from the end of the jet in a process that involves the formation of a long ligament. The flow rate and the electric current carried by the stream of droplets emitted in this mode are determined by the flow and the transfer of charge in the attached drop, in particular in a small region around its tip and in a leading stretch of the jet, where the solution is nearly stationary despite the transient character of the jet further downstream. A simplified analysis of the stationary regions is carried out to elucidate the effects of the physical properties of the liquid (electrical conductivity, permittivity, viscosity, and surface tension), the volume of the drop, and the strength of the applied field. For high electrical conductivities and applied fields well above its critical value, the electrical and viscous stresses are large compared to surface tension stresses, and their balance gives a flow rate proportional to the square of the applied field. The electric current is then that of a stationary electrified jet fed with this flow rate.
\end{abstract}

DOI: 10.1103/PhysRevE.78.016314

PACS number(s): 47.65.-d, 47.15.G-, 47.55.df

\section{INTRODUCTION}

The dynamics of an electrically neutral drop in a uniform electric field and of an electrically charged drop in a fieldfree region are classical problems that have been investigated for over a century. Applications include electrified cloud drops [1,2], processes involving electrostatic spraying [3,4], in particular in connection with the mass spectrometry of biomolecules [5], and ink-jet printing, among others. Rayleigh [6] determined the maximum charge that a spherical drop of a conducting liquid suspended in a dielectric fieldfree medium can hold as a function of its radius and the surface tension of the liquid. When this maximum charge is approached or exceeded, the drop becomes unstable and undergoes a Coulombic fission whereby it loses a fraction of its mass that may range from less than $0.1 \%$ to $30 \%$, and a fraction of its charge that may range from $10 \%$ to over $80 \%$; see Li et al. [7] and references therein. Fernández de la Mora [8] points out two different modes of Coulombic fission; a rough fission mode in which the drop divides into a few fragments of similar sizes [9-12], and a fine fission mode that proceeds via the formation of a conical tip and the ejection of a fine transient jet that produces many daughter drops much smaller than the parent drop [13-17]. Somewhat imprecisely, the first mode is associated with apolar liquids of low electrical conductivity, and the fine fission mode is typical of highly conducting liquids.

The tip of a drop in the fine fission mode resembles the conical meniscus of an electrospray in the cone-jet mode $[18,19]$, in which, under the action of an externally applied field, a stationary, supported, and continuously fed meniscus becomes conical and ejects a thin jet that eventually breaks into a spray of charged drops. This similarity led Fernández de la Mora [8] to propose that, if the characteristic time of formation of the jet in a Coulombic fission is short compared with the duration of the fission process, then the size of the daughter drops and the electric current they carry should be governed by the laws of stationary cone-jets for highly conducting liquids [4,20]. Fernández de la Mora [4] shows that the condition on the times can be easily satisfied with liquids of low viscosity, but not with the triethylene glycol used in some of the experiments of Li et al. [7], among others. Computations carried out by Betelú et al. [21] for liquids of infinite conductivity charged to the Rayleigh limit suggest that, in the absence of inertia, the surface develops a conical tip in a finite time, but with a semiangle smaller than the Taylor angle of $49.3^{\circ}$ [22] expected for a stationary cone-jet.

An isolated neutral drop of a conducting liquid is symmetrically elongated by a uniform electric field. The drop reaches a state of equilibrium with a finite elongation if the strength of the field is smaller than a critical value whose square is proportional to the surface tension of the liquid and inversely proportional to the product of the permittivity of the surrounding medium and the initial radius of the drop. The elongation of the drop at equilibrium and the critical electric field have been computed using a spheroidal approximation for the shape of the surface together with a balance of energy or of electric, pressure, and surface tension forces [22-24], or numerically [25-27]. Above the critical field, the electric stress acting on the surface of the drop disrupts it in a manner that depends on the physical properties of the liquid and the surrounding medium (see Ref. [28] and references therein). In vacuum or in a dielectric gas, which are the cases of interest here, the possible modes of evolution of a neutral drop are analogous to the rough and fine fission modes of a charged drop. The second of these modes, which is typical of liquids of high conductivity, has been investigated by Grimm and Beauchamp [29] for $225 \mu \mathrm{m}$ drops of methanol in air. In their visualizations, these authors observe a continuous elongation of the drop following the application of the field, until two conical tips 
develop and jets are ejected from the two poles of the drop after a time that depends on the volume of the drop and decreases when the strength of the applied field increases. The two jets emit charges of opposite polarities and very little mass. They do not induce a net charge in the drop, and therefore the duration of the process may be large compared to the duration of a Coulombic fission of a charged drop. Oddershede and Nagel [30] investigated the related problem of the development of a spout singularity and a jet at the surface of a liquid layer subjected to a high electric field normal to the surface. On the basis of their experimental results, these authors propose a power-law scaling for the final steps of the evolution leading to the singularity.

Reznik et al. [31] studied numerically and experimentally the first stages of the process for a drop attached to a plane electrode which is abruptly charged to a constant voltage relative to another parallel electrode. Their numerical computations for a liquid of infinite conductivity and a viscositydominated flow show that the attached drop develops a single conical tip with a semiangle of about $30^{\circ}$, not unlike that of Betelú et al. [21], or sheds a large blob of liquid if its initial volume is higher than a certain value. These results have been recently extended by Collins et al. [32]. Using the leaky dielectric model $[33,34]$ for a layer of a relatively apolar liquid of low electrical conductivity, these authors numerically describe the formation of a jet from the protrusion that appears at the surface following the application of the field and compute the evolution of the jet until a small droplet detaches from its tip. They analyze the crucial role played by the electric shear at the surface of the developing jet, and the viscous transfer of momentum to the bulk of the liquid. They find that the size of the detaching droplet is proportional to the product of the viscosity and the dielectric constant of the liquid, and inversely proportional to the cubic root of the product of its electrical conductivity and its surface tension.

In this paper, the process of formation of a jet and emission of small charged droplets from a parent drop attached to a plane electrode is further analyzed for liquids of high electrical conductivity in the framework of the leaky dielectric model. The effect of the inertia of the liquid, which was taken into account by Collins et al. [32], will be left out here. This approximation is bound to fail away from the drop for long jets, but it is admissible in the attached drop and the beginning of the jet for small drops and very viscous liquids. It brings in noticeable simplifications to the numerical treatment and the analysis of the results, which allows one to study liquids of moderately high electrical conductivity, for which the emitted jet is very thin, and long times in the evolution of the jet. In the absence of inertia, the detachment of droplets is found to involve the formation of a long ligament and is not periodic. However, the numerical results for moderately high electrical conductivities show that, when the droplets detach continuously from the end of a jet, the flow and the electric field rapidly become stationary in a small region around the tip of the attached drop and in a leading region of the jet, which determine the electric current emitted by the drop. A current transfer region analogous to the charge relaxation region of Refs. $[4,20]$ is identified at the beginning of the jet where the finite conductivity of the liquid limits the conduction current that transfers charge to the surface. Qualitative scaling laws for the flow rate, the electric current, and the radius of the jet are worked out in limited ranges of the parameters of the problem.

\section{FORMULATION}

A drop of volume $V^{*}$ of a liquid of viscosity $\mu$, surface tension $\gamma$, electrical conductivity $K$, and permittivity $\epsilon_{0} \epsilon$, where $\epsilon_{0}$ is the permittivity of the vacuum or the dielectric gas surrounding the drop, stands on a plate electrode. At a certain time the electrode is charged to a high voltage relative to another distant parallel plate, which leads to a uniform electric field $E_{\infty}^{*}$ far from the drop. The electric field causes electric stresses at the surface [right-hand sides of Eqs. (4b) and $(4 \mathrm{c})$ below] that elongate the drop against the restoring effect of the surface tension. The flow of the liquid is assumed to be dominated by viscous forces, and the radius $a$ of the wetted circle on the electrode is assumed to remain constant during the evolution of the drop.

The electric fields in the liquid and the surrounding medium are of the form $\boldsymbol{E}^{l}=-\boldsymbol{\nabla} \varphi^{l}$ and $\boldsymbol{E}=-\boldsymbol{\nabla} \varphi$, respectively, where $\varphi^{l}$ and $\varphi$ are the electric potentials in the liquid and outside. The electric field $\boldsymbol{E}^{l}$ leads to a conduction current density $\boldsymbol{j}=K \boldsymbol{E}^{l}$, and conservation of charge requires $\boldsymbol{\nabla} \cdot \boldsymbol{j}=0$ in the absence of net charge in the bulk of the liquid. The electric potentials satisfy Laplace's equations $\nabla^{2} \varphi=\nabla^{2} \varphi^{l}=0$ with the boundary conditions $\epsilon_{0}\left(E_{n}-\epsilon E_{n}^{l}\right)=\sigma$ and $E_{t}=E_{t}^{l}$ at the surface of the liquid (Landau and Lifshitz [35]), $\varphi=\varphi^{l}=0$ at the electrode, and $\nabla \varphi=-E_{\infty}^{*} i$ far from the drop. Here subscripts $n$ and $t$ denote the components of the fields normal and tangent to the surface of the liquid (with $\boldsymbol{n}$ pointing away from the liquid), $i$ is a unit vector normal to the electrode, and $\sigma$ is the density of free surface charge, which satisfies the conservation equation (Saville [34])

$$
\frac{D \sigma}{D t}=K E_{n}^{l}+\sigma \boldsymbol{n} \cdot \nabla \boldsymbol{v} \cdot \boldsymbol{n}
$$

at the surface. Here $v$ is the velocity of the liquid and $D \sigma / D t=\partial \sigma / \partial t+\boldsymbol{v} \cdot \nabla \sigma$ is the material derivative at the surface. Equation (1) expresses that the surface charge is convected by the liquid, with its density increasing due to the net charge that conduction brings to the surface per unit area and time (first term on the right-hand side of the equation) and decreasing due to stretching of the surface $(\boldsymbol{n} \cdot \nabla \boldsymbol{v} \cdot \boldsymbol{n}$ in the second term on the right-hand side is the negative of the straining rate of a material element of the surface; see, e.g., Batchelor [36]). The balance of surface charge accumulation and conduction toward the surface, $\sigma / t_{e}^{*} \sim K E_{n}^{l}$, with $\sigma$ $\sim \epsilon_{0} E_{n} \sim \epsilon_{0} \epsilon E_{n}^{l}$ from the boundary condition for the normal electric field, determines the characteristic time $t_{e}^{*}=\epsilon_{0} \epsilon / K$ required for the surface charge to approach its equilibrium value in a quiescent surface $\left(\sigma_{e q}=\epsilon_{0} E_{n}\right)$ and screen the liquid from the applied field. The relaxation time $t_{e}^{*}$ is a combination of physical properties of the liquid only.

The Reynolds number $\operatorname{Re}=\rho v_{c} a / \mu=\rho \gamma a / \mu^{2}$, where $\rho$ is the density of the liquid and $v_{c}=\gamma / \mu$ is a characteristic velocity determined by an order-of-magnitude balance of vis- 
cous and surface tension stresses, is assumed to be very small, so that the effect of the inertia of the liquid can be neglected. The condition $\operatorname{Re}<1$ requires $a<10 \mu \mathrm{m}$ for ethylene glycol, $a<200 \mu \mathrm{m}$ for palm oil, and $a<10 \mathrm{~mm}$ for glycerine, and droplet sizes well below these values should be used to ensure that the effect of the inertia of the liquid is small also in a leading region of the jet and for high values of the applied electric field (see Sec. III C below).

Dimensionless variables are introduced using the radius $a$ of the wetted circle, the viscous-capillary velocity $v_{c}=\gamma / \mu$, and the electric field $E_{c}=\gamma^{1 / 2} \epsilon_{0}^{-1 / 2} a^{-1 / 2}$ as units of length, velocity, and electric field. The electric potentials, density of surface charge, and electric current are measured with $E_{c} a$, $\epsilon_{0} E_{c}$, and $\epsilon_{0} E_{c} v_{c} a$, respectively. Denoting by $f(\boldsymbol{x}, t)=0$ the surface of the liquid, which is a material surface to be determined as part of the solution, the governing equations and boundary conditions take the form

$$
\boldsymbol{\nabla} \cdot \boldsymbol{v}=0, \quad 0=-\nabla p+\nabla^{2} \boldsymbol{v}, \quad \nabla^{2} \varphi^{l}=0
$$

in the liquid, for $f(\boldsymbol{x}, t)<0$,

$$
\nabla^{2} \varphi=0
$$

outside the liquid, for $f(\boldsymbol{x}, t)>0$,

$$
\begin{gathered}
\frac{D f}{D t}=0, \\
-p+\boldsymbol{n} \cdot \tau^{\prime} \cdot \boldsymbol{n}+\boldsymbol{\nabla} \cdot \boldsymbol{n}=\frac{1}{2}\left(E_{n}^{2}-\epsilon E_{n}^{l^{2}}\right)+\frac{1}{2}(\epsilon-1) E_{t}^{2}, \\
\boldsymbol{t} \cdot \boldsymbol{\tau}^{\prime} \cdot \boldsymbol{n}=\sigma E_{t}, \\
E_{n}-\epsilon E_{n}^{l}=\sigma, \\
E_{t}=E_{t}^{l}, \\
\frac{D \sigma}{D t}=\Lambda E_{n}^{l}+\sigma \boldsymbol{n} \cdot \boldsymbol{\nabla v} \cdot \boldsymbol{n}
\end{gathered}
$$

at the surface, $f(\boldsymbol{x}, t)=0$,

$$
\begin{gathered}
v=0, \\
\varphi=\varphi^{l}=0
\end{gathered}
$$

at the electrode $(x=0)$, and

$$
\nabla \varphi=-E_{\infty} i
$$

far from the electrode. Here $p$ is the dimensionless pressure of the liquid referred to the pressure of the surrounding medium; $\tau^{\prime}=\boldsymbol{\nabla} \boldsymbol{v}+(\boldsymbol{\nabla v})^{T}$ is the dimensionless viscous stress tensor; $\boldsymbol{n}$ and $\boldsymbol{t}$ are unit vectors normal and tangent to the surface; and $x$ is the dimensionless distance to the electrode. Equations (4b) and (4c) are balances of stresses normal and tangent to the surface. The right-hand sides of these equations are the components of the electric stress normal and tangent to the surface, denoted $\tau_{n}^{e}$ and $\tau_{t}^{e}$ in what follows (Landau and Lifshitz [35], Saville [34]).
The solution of the problem depends on the dimensionless parameters

$$
V=\frac{V^{*}}{a^{3}}, \quad E_{\infty}=\frac{\epsilon_{0}^{1 / 2} a^{1 / 2} E_{\infty}^{*}}{\gamma^{1 / 2}}, \quad \epsilon, \quad \Lambda=\frac{\mu K a}{\epsilon_{0} \gamma},
$$

where $V$, the dimensionless volume of the drop, appears in the initial conditions. Initially the drop is taken to be a spherical cap with $\sigma=0$. Attention is given primarily to cases with large values of the dimensionless electrical conductivity $\Lambda$.

Axisymmetric solutions of Eqs. (2)-(7) have been computed using standard boundary elements methods to solve the Laplace and Stokes equations (2) and (3) and a secondorder Runge-Kutta method to advance the evolution equations (4a) and (5c). The dimensionless distance to the symmetry axis and the radius of the cross section are denoted $r$ and $r_{s}(x, t)$ in what follows.

\section{RESULTS AND DISCUSSION}

\section{A. Transient computations}

The critical value of the dimensionless electric field $\left(E_{\infty_{c}}\right)$ below which a hydrostatic solution exists is a decreasing function of the dimensionless volume of the drop. It is $E_{\infty}$ $\approx 0.86$ for $V=0.9$, which is about the volume of a drop shaped as a Taylor cone, and $E_{\infty_{c}} \approx 0.56$ for $V=2$. The equilibrium shapes do not depend on $\epsilon$ and $\Lambda$.

The elongation of the drop increases continuously with time when $E_{\infty}>E_{\infty}$. In agreement with the numerical results of Reznik et al. [31] for liquids of infinite conductivity, the drop is seen to develop a nearly conical shape around its tip, with a semiangle smaller than the Taylor angle. The liquid speeds up in a region of decreasing size around the tip, $x$ $=x_{\text {tip }}(t)$. The time evolution of $x_{\text {tip }}$ is shown in the inset of Fig. 3 below for a sample case. A power law $\left(x_{S}-x_{\text {tip }}\right) \propto\left(t_{S}\right.$ $-t)^{\alpha}$ can be fitted to the final steps of this evolution, where $x_{S}$ and $t_{S}$ denote the location and time of the apparent singularity $\left(x_{S} \approx 1.95\right.$ and $t_{S} \approx 6.2$ for the case of Fig. 3$)$ and the exponent $\alpha$ is about 0.5 . This value is similar to the one found by Betelú et al. [21] for an isolated drop charged to the Rayleigh limit, but somewhat higher than the experimental value 0.42 reported by Oddershede and Nagel [30], which might be due to the effect of the inertia of the liquid in the experiments.

The evolution departs from a power law when a small patch of liquid protrudes from the rounded tip. In the computations, this patch becomes a jet that eventually emits small droplets, as illustrated in Fig. 1 for a sample case. The radius of the jet decreases when the dimensionless conductivity $\Lambda$ increases.

Figure 2 shows the distributions of electric field, surface charge, and electric and surface tension stresses at the surface of the drop and the jet at two instants of time. The left-hand side panels are for a time slightly after the detachment of a droplet, and the right-hand side panels are for a time before the detachment of the following droplet. As can be seen, the conditions in the attached drop and in a drop-tojet transition region (around $x=1$ in the figure) are nearly 


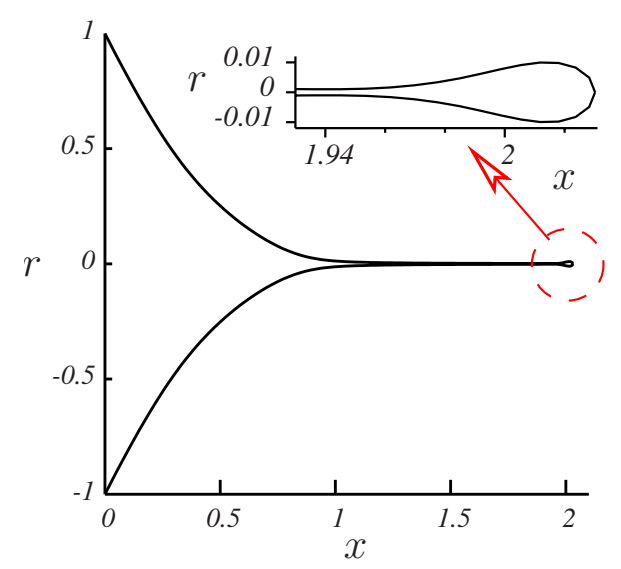

FIG. 1. (Color online) Shape of the surface at $t=3.08860$, with a droplet about to detach. Values of the parameters are $V=0.8, E_{\infty}$ $=1.25, \epsilon=20$, and $\Lambda=10^{3}$.

independent of time. The flow rate and the electric current entering the jet are also independent of the transient flow in the jet further downstream. The dimensionless surface charge coincides with the dimensionless normal electric field in the attached drop, where the residence time of the liquid is large compared with the electric relaxation time $\left(t_{e}=\epsilon / \Lambda\right.$ in dimensionless variables), but not in the drop-to-jet transition region, where both times are of the same order and the surface charge cannot follow the evolution of the normal field, though both variables go through a maximum and decrease in the jet. These results agree with the general picture put forward by Fernández de la Mora and Loscertales [20] for electrosprays of highly conducting liquids working in the stationary cone-jet mode with small flow rates.

The surface tension and normal electric stress balance each other in the drop, where the electric shear is small (but see comments at the end of Sec. III B and in Sec. III C 1 below for large values of $E_{\infty}$ ). The normal electric stress is larger than the surface tension in the transition region, leading to the outward curvature of the surface that forms the jet,
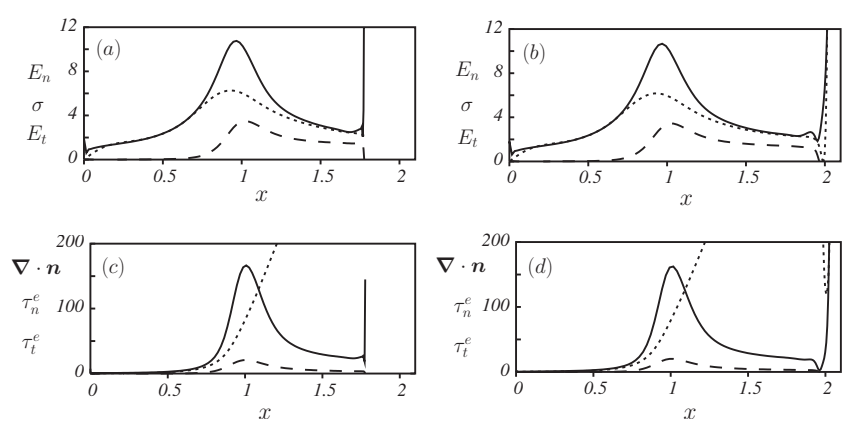

FIG. 2. (a) and (b) Surface distributions of normal electric field (solid), tangent electric field (dashed), and density of surface charge (dotted) as functions of streamwise distance for $t=3.08763$ [in (a), after the detachment of a droplet] and $t=3.08860$ [in (b), before the detachment of the following droplet]. (c) and (d) Surface distributions of normal electric stress (solid), tangent electric stress (dashed), and surface tension stress (dotted) at the same instants of time as in (a) and (b). Values of the parameters are $V=0.8, E_{\infty}$ $=1.25, \epsilon=20$, and $\Lambda=10^{3}$.

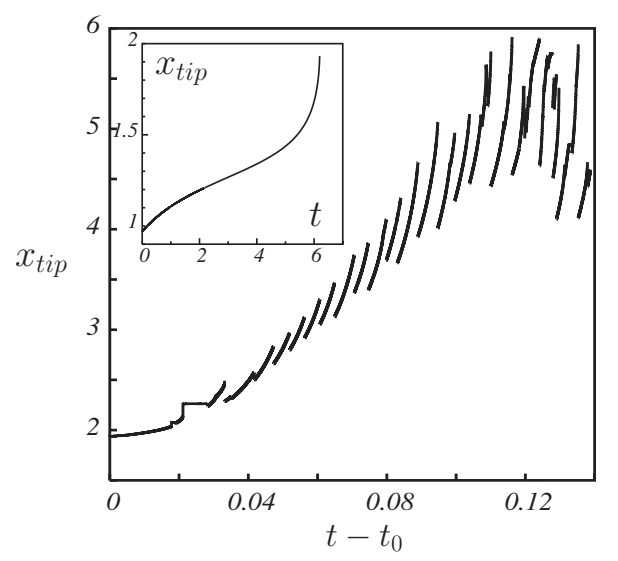

FIG. 3. Time evolution of the tip of the jet for $V=2, E_{\infty}=0.6$, $\epsilon=20$, and $\Lambda=2 \times 10^{3}$. The main figure is a blowup of a small range of $t$ following the smooth elongation shown in the inset. $t_{0}=6.195$. Discontinuities mark the detachment of droplets.

but the situation reverses further downstream, where the inward surface tension stress increases monotonically with streamwise distance and soon becomes the dominant stress to be balanced by the pressure and viscous stresses of the liquid on the surface. This suggests that the final stages of drop pinchoff are not much affected by local electric forces. The capillary pinchoff of a very viscous liquid in the absence of electric forces is known to be a complex process that involves the formation of a long filament [37], but the fine details of this process are not captured by the present computations. Here, following previous work [38], a droplet is taken to detach when the minimum radius of the jet becomes smaller than a certain cutoff value of the order of a thousandth of the radius of the base of the attached drop. The jet is then cut at its minimum radius and the surface is artificially rounded to continue the computation until another droplet forms and detaches from the jet.

The evolution of the jet has been followed in this manner through a number of detachments. The loss of volume of the drop at each detachment is very small, of about $1.5 \times 10^{-5}$ for the case shown in Fig. 1. The distance that the tip of the jet travels between successive detachments is at first larger than the length of the detached droplet, so that the tip follows the sawtooth trajectory illustrated in Fig. 3, with a continuous increase of the average length of the jet. The tip may undergo a large number of detachments, and the average length of the jet may increase to fairly large values, before the process stabilizes. This poses severe restrictions on the precision of the computations. The time evolution is still not periodic after the average length of the jet levels off, and it stays nonperiodic even when the small volume lost at each detachment is artificially added back to the attached drop.

\section{B. Stationary computations}

The preceding computations for moderately large values of $\Lambda$ show that the flow and the electric field are very nearly stationary around the tip of the attached drop and in a leading stretch of the jet, both before the average length of the jet stabilizes and in the continuous emission mode that is estab- 
lished afterwards. These regions are of interest because, as will be seen in Sec. III C below, they determine the electric current carried by the jet. Being quasistationary, they can be subject to the same type of analysis used for a strictly stationary cone-jet [4].

It should be noted that the continuous emission mode illustrated by these computations is not the only mode by which small droplets can be emitted from an electrified meniscus. There are other emission modes that exhibit a cyclic depletion and buildup of part of the meniscus, whose tip is no longer quasistationary; see Refs. $[18,19,39]$ and the videos in the supplementary material of Ref. [40]. A case in point is the set of pulsating modes of a nanoelectrospray. A nanoelectrospray consists of a capillary drawn to $1-2 \mu \mathrm{m}$ exit diameter and loaded with a liquid that may be sprayed into drops in the 100-nm-diameter range at flow rates of the order of $3 \times 10^{-10} \mathrm{~m}^{3} / \mathrm{s}$ under the action of a high voltage applied between the capillary and another electrode $[41,42]$. A nanoelectrospray may work without any pressure applied to drive the liquid, whose flow rate is dictated by the applied voltage, the needle geometry, and the orifice diameter. It may function in various pulsating modes $[43,44]$, which typically give way to a stable cone-jet mode when the voltage is increased. Similar pulsating modes might exist for a drop attached to a plate electrode, though some features of the pulsating modes of a nanoelectrospray seem to be specific and connected to the hydraulic resistance of the narrow capillary of this device [43]. Pulsating modes will not be discussed here.

Turning back to the continuous emission mode, it can be seen that the distribution of conduction current in the bulk of the attached drop is quasistationary when $\Lambda$ is large. Since $\boldsymbol{\nabla} \cdot \boldsymbol{j}=0$, the difference between the electric current that enters the base of the drop by conduction from the electrode and the conduction current that reaches the quasistationary region around the tip is equal to the rate at which conduction brings charge to the surface of the drop. This charge may accumulate at the surface or be convected by the flow, leading to a surface convection current that adds to the conduction current in the bulk of the liquid. However, the convection current is small compared to the conduction current upstream of the tip (see Fig. 8 and the discussion in Sec. III C below), and the rate of accumulation at the surface tends to zero in a time of the order of the electric relaxation time $(\epsilon / \Lambda$ in dimensionless variables) required for the surface charge to achieve equilibrium and render $E_{n}^{l}$ negligible in the drop. Here this time is short compared to the mechanical time of evolution of the drop, and therefore the drop transfers conduction current unaltered from the electrode to the quasistationary region around its tip, where it is effectively transferred to the surface.

The flow needs not be quasistationary in the bulk of the drop, but its slow time variation is not expected to affect the dynamics of the small quasistationary regions of interest. Here the following approximations are used to avoid computing both the evolution of this flow as the drop is slowly consumed and the fast evolution of the flow in the transient region of the jet. First, the jet is artificially truncated at a constant distance from the electrode, which is sufficiently large to ensure, through numerical tests, that the solution in the regions of interest is insensitive to the truncation. This approximation has been used before in the numerical computations of continuously fed jets $[45,46]$. Second, the flow rate crossing the far end of the truncated jet is artificially reinjected into the drop, in order to keep the volume of liquid constant. This approximation makes a stationary solution possible in certain regions of the parameter space. As far as the quasistationary regions are concerned, solutions for different values of the volume of liquid should be nearly equivalent to solutions for different times during the consumption of a real drop. Reinjection of liquid into the drop makes the model resemble the meniscus of a nanoelectrospray, in which capillary forces draw liquid from the capillary tube to resupply the liquid lost at the apex. The analogy has been used elsewhere [47] to analyze the related but hydrodynamically simpler problem of the meniscus of an ion source. However, this analysis shows that the pressure drop in the feeding tube, which is not accounted for here, may play a role in the stability and transient response of such systems.

A stationary solution with a truncated jet is found for values of $E_{\infty}$ above a certain minimum that depends on $V, \epsilon$, and $\Lambda$. When the minimum $E_{\infty}$ is approached from above, the stationary surface develops a neck that heralds pinchoff at a section between the attached drop and the jet. Depending on the values of the parameters, this minimum may be smaller or larger than the critical value $E_{\infty}$ at which a rounded hydrostatic drop ceases to exist [see inset of Fig. 4(a)]. The first case leads to hysteresis and multiplicity in a range of $E_{\infty}$ that depends on $V, \epsilon$, and $\Lambda$. In the second case no stationary solution is found in a range of $E_{\infty}$ where pulsating modes could develop, though this possibility is not explored here.

The stationary flow rate entering the jet is shown in Fig. 4. Contrary to the case of the continuously fed meniscus of an electrospray [4], in which it is given at the outset, the flow rate is determined here as part of the solution. It increases with $E_{\infty}$, as could be expected from the increase of the electric stresses acting on the drop. The flow rate also increases with $\Lambda$ at moderate values of this parameter, though it seems to level to an asymptotic value when $\Lambda$ is increased keeping the other parameters constant [Fig. 4(b)].

The electric field acting on the attached drop is nearly normal to its surface. The normal field $E_{n}$ (at the outer side of the surface) has the same appearance as in Fig. 2 above. It increases on approaching the tip of the drop, reaches a maximum at the beginning of the jet, and then decreases with streamwise distance. The component of the electric field tangent to the surface, $E_{t}$, rises to a shallow maximum at about the same point as $E_{n}$ or slightly downstream of it, and falls toward $E_{\infty}$ in the jet. The maxima of $E_{n}$ and $E_{t}$ increase and shift slightly upstream when $E_{\infty}$ increases [Fig. 5(a)], and they increase and shift slightly downstream when $\Lambda$ increases [Fig. 5(b)]. The dotted curve of Fig. 5(b) shows the electric field on the surface of a liquid of infinite conductivity. The field for finite values of the dimensionless conductivity $\Lambda$ branches off this curve after following it in a leading region of the surface whose size increases with $\Lambda$. The rise of the tangent field is also postponed when $\Lambda$ increases [dashed curves in Fig. 5(b)], keeping the surface nearly equipotential 

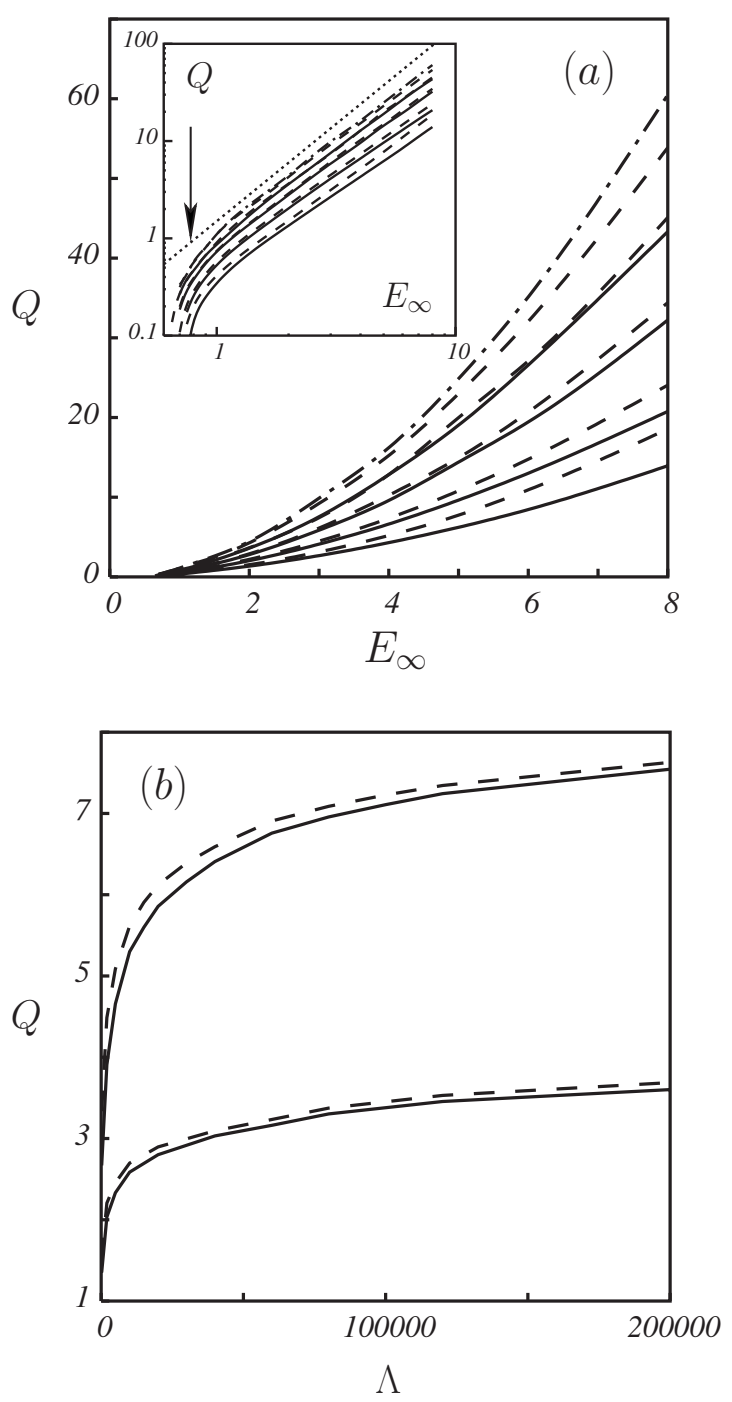

FIG. 4. (a) Dimensionless flow rate as a function of $E_{\infty}$ for $V$ $=0.9, \epsilon=2$ (solid) and 20 (dashed), and $\Lambda=2 \times 10^{2}, 2 \times 10^{3}, 2$ $\times 10^{4}$ and $2 \times 10^{5}$, increasing from bottom to top. The two upper curves are for $\Lambda=2 \times 10^{6}$, with $\epsilon=20$ (dashed line) and 200 (dashand-dot line). The dotted line in the inset has slope 2 . The vertical arrow marks the critical field $E_{\infty}$ above which a rounded hydrostatic drop does not exist. (b) Dimensionless flow rate as a function of $\Lambda$ for $V=0.9, \epsilon=2$ (solid) and 20 (dashed), and $E_{\infty}=2$ (lower curves) and 3 (upper curves).

in the leading region. The end of this region is marked by a decrease in the rate at which the radius of the jet decreases with streamwise distance, which is very large for a liquid of infinite conductivity.

The radius of the jet at the position of maximum $E_{n}$ (Fig. 6) increases with $E_{\infty}$ and decreases when $\Lambda$ or $\epsilon$ are increased, though the dependence on $\epsilon$ is moderate and felt mostly for large values of $E_{\infty}$. The increase of the radius with $E_{\infty}$ reflects that the effect of the electric stresses on the drop, which tends to increase the flow rate, overcomes the effect of the electric shear on the jet, which tends to speed up the liquid and causes the radius to decrease when $E_{\infty}$ is increased at constant flow rate (see, e.g., Ref. [45]).

The density of surface charge $\sigma$ follows the evolution of $E_{n}$. Electric conduction brings electric charge to the surface
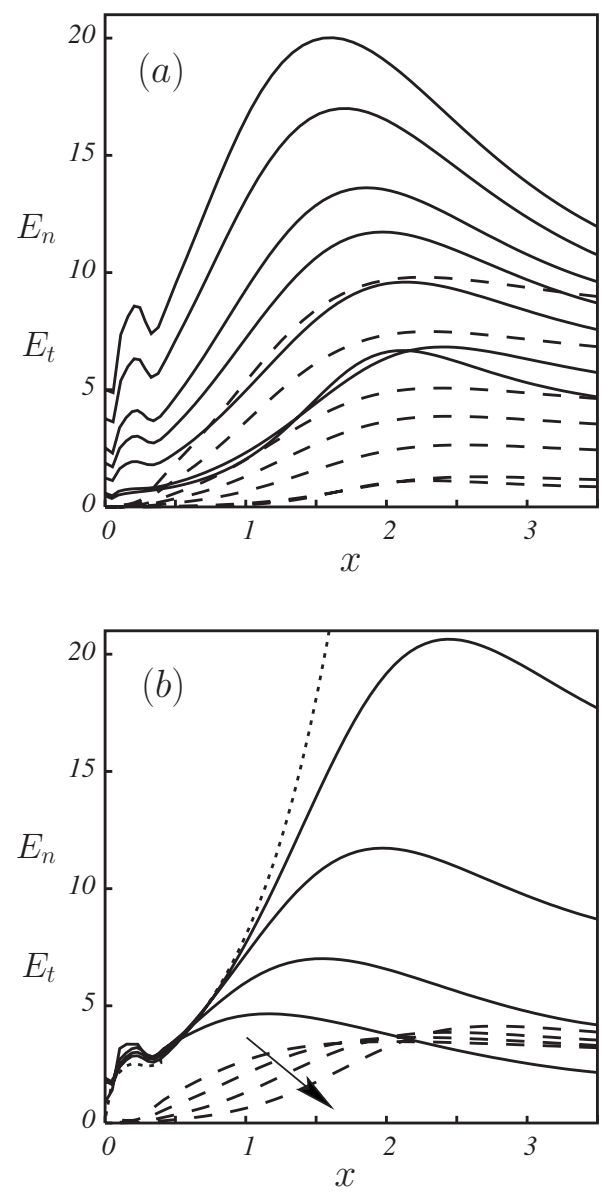

FIG. 5. Normal electric field (solid line) and tangent electric field (dashed line) as functions of streamwise distance for $V=0.9$ and $\epsilon=20$. In (a), $E_{\infty}=0.7,0.9,2,3,4,6$, and 8 (increasing from bottom to top), and $\Lambda=2 \times 10^{4}$. In (b), $E_{\infty}=3$ and $\Lambda=2 \times 10^{2}, 2$ $\times 10^{3}, 2 \times 10^{4}$ and $2 \times 10^{5}$, increasing from bottom to top for $E_{n}$, and as indicated by the arrow for $E_{t}$. The dotted curve is the normal electric field for a liquid of infinite conductivity.

of the drop and the leading part of the jet, where the normal electric field at the liquid side of the surface $\left(E_{n}^{l}\right.$, not displayed) also reaches a maximum. The density of surface charge decreases further downstream due to the continuous stretching of the surface. The ratio $\epsilon E_{n}^{l} / E_{n}$ of the electric displacements at the two sides of the surface is a measure of the departure of the surface charge from the equilibrium condition $\sigma_{e q}=E_{n}$; see Eq. (5a). The maximum value of $\epsilon E_{n}^{l} / E_{n}$, which is attained in the region of maximum $E_{n}$, is shown in Fig. 7 for different values of the parameters. This ratio increases with $E_{\infty}$ and $\epsilon$ and decreases when $\Lambda$ increases.

The electric current carried by the jet is made of the surface current due to the convection of surface electric charge, $I_{s}=2 \pi r_{s} \sigma v_{s} r_{s}$ where $v_{s}$ is the velocity of the liquid at the surface, and the conduction current in the bulk of the liquid, $I_{b}=\pi \Lambda \int_{0}^{r_{s}} r E_{x}^{l} d r$ where $E_{x}^{l}$ is the axial component of the electric field in the liquid. The axial evolution of $I_{s}$ and $I_{b}$ is shown in Fig. 8 for a few cases. The total current $I=I_{s}+I_{b}$ is a constant independent of $x$ that increases with $E_{\infty}$ and with $\Lambda$ (Fig. 9). It increases faster than linearly with $E_{\infty}$ because not only the axial field $E_{x}^{l}$ but also the radius of the jet in- 

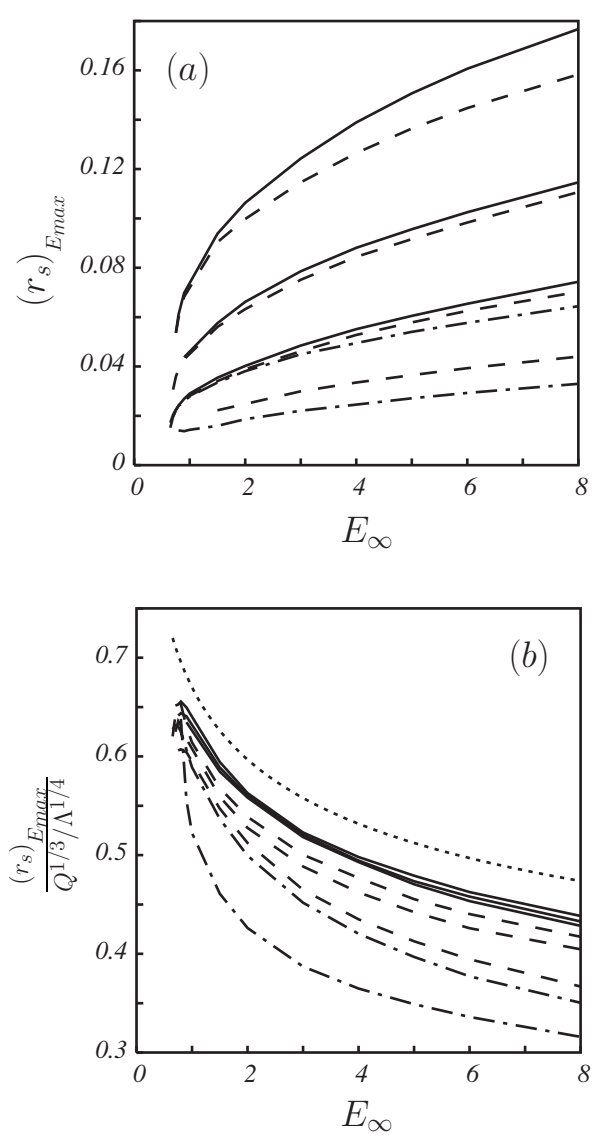

FIG. 6. (a) Radius of the jet at the position of maximum $E_{n}$ as a function of $E_{\infty}$ for $V=0.9, \epsilon=2$ (solid line) and 20 (dashed line), and $\Lambda=2 \times 10^{3}, 2 \times 10^{4}$ and $2 \times 10^{5}$, increasing from top to bottom. The lower dashed curve is for $\Lambda=2 \times 10^{6}, \epsilon=20$. The dash-and-dot curves are for $\epsilon=200, \Lambda=2 \times 10^{5}$ (upper), and $2 \times 10^{6}$ (lower). (b) Same data with the radius scaled with $Q^{1 / 3} / \Lambda^{1 / 4}$. The solid curves, for $\epsilon=2$ and $\Lambda=2 \times 10^{3}, 2 \times 10^{4}$, and $2 \times 10^{5}$, nearly collapse. The dashed curves are for $\epsilon=20$ and the same values of $\Lambda$, increasing from bottom to top. The dash-and-dot curves are for $\epsilon=200$ and $\Lambda=2 \times 10^{5}$ (upper) and $2 \times 10^{6}$ (lower). The dotted curve in the upper part of the figure is $0.67 / E_{\infty}^{1 / 6}$.

creases with $E_{\infty}$ in the region where $I_{b}$ makes a noticeable contribution of the current. Similarly, $I$ increases less than linearly with $\Lambda$ because the radius of the jet decreases when $\Lambda$ increases. The current decreases slightly with $\epsilon$, whose main effect is to lower the rate at which $I$ increases with $E_{\infty}$ at large values of this parameter.

The electric stresses normal and tangent to the surface [right-hand sides of Eqs. (4b) and (4c)] also reach their maxima at the beginning of the jet; see Figs. 2(c) and 2(d). The normal stress is large compared to the tangent stress, and both increase with $E_{\infty}$ and $\Lambda$. When $\epsilon=20$ or above, the term $\frac{1}{2}(\epsilon-1) E_{t}^{2}$ on the right-hand side of Eq. (4b) makes a large contribution to $\tau_{n}^{e}$ in a region around its maximum, whereas this term is outweighed by $\frac{1}{2}\left(E_{n}^{2}-\epsilon E_{n}^{l^{2}}\right)$ when $\epsilon=2$. The normal electric stress becomes large compared to the normal stress due to surface tension when $E_{\infty}$ increases. The ratio $\boldsymbol{\nabla} \cdot \boldsymbol{n} / \tau_{n}^{e}$ rapidly becomes small in the attached drop and in a leading region of the jet, though it increases with streamwise distance in the jet.

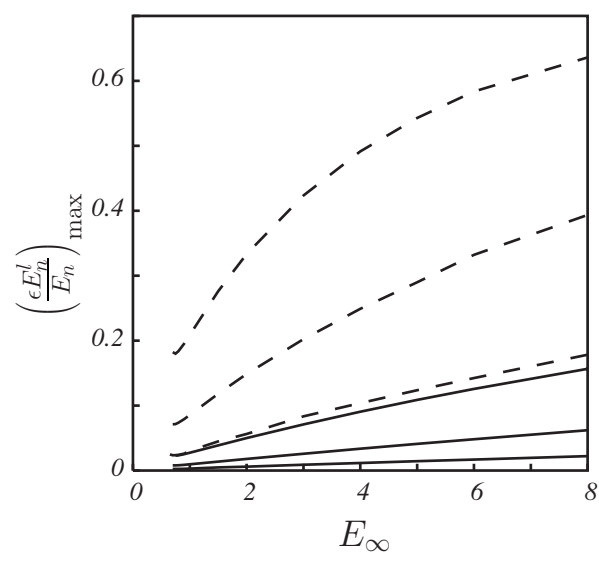

FIG. 7. Maximum value of $\epsilon E_{n}^{l} / E_{n}$ on the surface as a function of $E_{\infty}$ for $V=0.9, \epsilon=2$ (solid line) and 20 (dashed line), and $\Lambda=2$ $\times 10^{3}, 2 \times 10^{4}$, and $2 \times 10^{5}$, increasing from top to bottom.

\section{Orders of magnitude}

\section{Flow rate}

The relative weakness of the surface tension stress for large values of $E_{\infty}$ suggests that the characteristic velocity of the liquid in the drop may be determined by a balance of electric and viscous stresses, which gives $v \sim E_{\infty}^{2}$ in dimensionless variables $\left(\mu v / a \sim \epsilon_{0} E_{\infty}^{*^{2}}\right.$ in dimensional variables). Since the numerical results do not show strong recirculation in the drop, the dimensionless flow rate entering the jet (nondimensionalized with $\gamma a^{2} / \mu$ ) should be $Q \sim E_{\infty}^{2}$. Surface tension plays a role for moderate values of $E_{\infty}$, and the flow rate also depends on the dimensionless conductivity $\Lambda$ when it is not sufficiently large for the radius of the jet to be small compared to the size of the drop. However, Fig. 4(b) shows that the flow rate becomes independent of $\Lambda$ for large values of this parameter, and Fig. 4(a) displays the predicted quadratic dependence of $Q$ on $E_{\infty}$ for moderately large values of $E_{\infty}$.

This qualitative estimate is at variance with the hydrostatic balance of surface tension and normal electric stress that is generally taken to prevail in the meniscus of an electrospray $[4,20]$ or in a charged drop undergoing a Coulombic fission [8] when the conductivity of the liquid is sufficiently high to assume that the size of the meniscus or the drop is effectively infinite at the scale of the radius of the jet, and therefore does not affect the dynamics of the jet. Fernández de la Mora [8] argues that, in these conditions, the flow rate carried by the jet emitted by an isolated charged drop should be of the order of the theoretical minimum at which a stationary cone-jet can be established. This minimum depends on the surface tension and the dielectric constant of the liquid (as well as on its viscosity and conductivity, in the case of a viscosity-dominated flow discussed here; see, e.g., Ref. [48]) but does not depend on the size of the drop, while the opposite is predicted by the estimate of the previous paragraph for large $E_{\infty}$. These differences might be attributed to the moderate values of the dimensionless conductivity used in the computations on which the estimate relies, which are limited by numerical constrains and are smaller than typical 

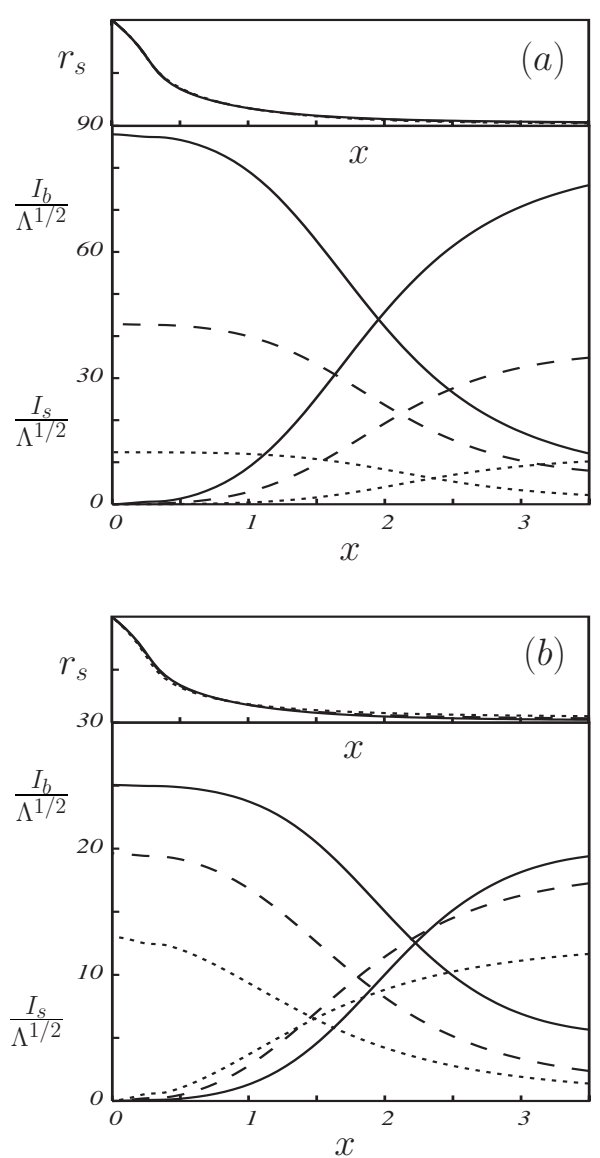

FIG. 8. Surface convection current (decreasing) and bulk conduction current (increasing) as functions of streamwise distance for $V=0.9$ and $\epsilon=20$. In (a) $E_{\infty}=2$ (dotted line), 4 (dashed line), and 6 (solid line), and $\Lambda=2 \times 10^{5}$. In (b) $E_{\infty}=3$ and $\Lambda=2 \times 10^{3}$ (dotted line), $2 \times 10^{4}$ (dashed line), and $2 \times 10^{5}$ (solid line). The meridional section of the surface is shown in the upper part of the figures.

experimental values. However, Fig. 4(b) shows no hint of drastic changes for larger values of $\Lambda$ and, despite the absence of a hydrostatic conical tip, a thin jet that breaks into a large number of small droplets places these numerical results nearer to the fine fission mode of polar conducting liquids [8] than to the rough fission mode in which large attached drops [31] or drops of apolar liquids of low electrical conductivity divide into a few fragments of similar sizes.

A hydrostatic drop with a conical tip seems to be possible only in a narrow range of values of $E_{\infty}$. For a given shape of the equipotential surface of the liquid featuring a Taylor cone in a region around its tip, the electric field in this region will be proportional to the inverse of the square root of the distance to the apparent vertex of the cone, but the strength of the field will be proportional to $E_{\infty}$, and thus the precise strength that is required to balance surface tension and electric stresses [22] will be attained only for a precise value of $E_{\infty}$. This strict condition may be relaxed somewhat when the ability of the surface away from the tip to adjust to variations of $E_{\infty}$, and the effect of the charge of the ejected jet on the electric field in the conical region of the surface, are taken into account; but it is unlikely that these mechanisms could lead to a stabilized Taylor cone in a wide range of $E_{\infty}$.
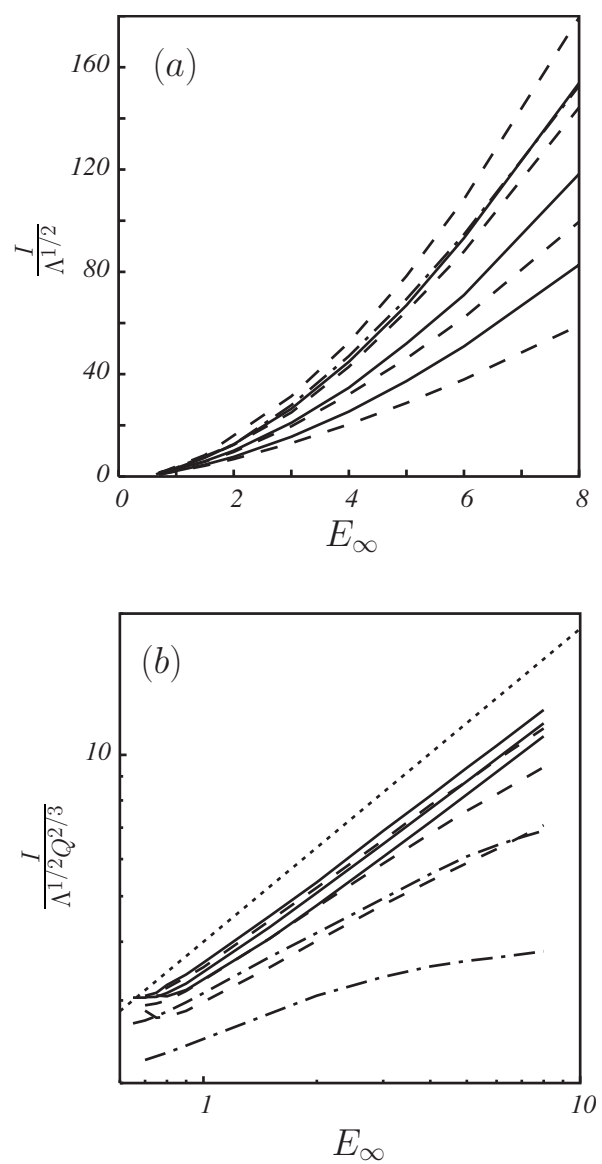

FIG. 9. (a) Electric current divided by $\Lambda^{1 / 2}$ as a function of $E_{\infty}$ for $V=0.9, \epsilon=2$ (solid line) and 20 (dashed line), and $\Lambda=2 \times 10^{3}$, $2 \times 10^{4}$, and $2 \times 10^{5}$, increasing from bottom to top. The two upper curves are for $\Lambda=2 \times 10^{6}$ and $\epsilon=20$ (dashed line) and 200 (dashand-dot line). (b) Same data with the current scaled with $\Lambda^{1 / 2} Q^{2 / 3}$. As in (a), $\Lambda=2 \times 10^{3}, 2 \times 10^{4}$, and $2 \times 10^{5}$ increasing from bottom to top for $\epsilon=2$ (solid line) and 20 (dashed line). The two dash-anddot curves are for $\epsilon=200$ and $\Lambda=2 \times 10^{4}$ (lower) and $2 \times 10^{5}$ (upper). The dotted line has slope $2 / 3$.

\section{Current transfer region}

Other features of the flow in the jet that are not directly connected with the determination of the flow rate can be qualitatively analyzed as for the jet of an electrospray. Let us first estimate the time that it would take for electrical conduction in the liquid to establish the condition of equilibrium of the surface charge $\left(\sigma=E_{n}\right)$, beginning with an initially uncharged surface $(\sigma=0)$, in a stretch of the jet of characteristic length $x$ and characteristic radius $r_{s} \ll x$. Being a slender body, the jet acts electrically as a line of charge, which induces an electric field whose radial and axial components at a distance of order $r_{s}$ from the axis satisfy $E_{r} / E_{x}=O\left(x / r_{s}\right)$ up to logarithms of $x / r_{s}$; see, e.g., Refs. [49,50]. The surface of the jet is an equipotential in the final equilibrium state, which means that the axial component of the induced field must oppose the applied field $E_{\infty}$ and balance it at the surface. This determines the order of the electric field normal to the surface as $E_{n} \sim E_{\infty} x / r_{s}$, which is also the order of the dimensionless density of surface charge at equilibrium, while the 
total surface charge in the stretch of the jet considered is $\mathcal{C}$ $\sim E_{n} r_{s} x \sim E_{\infty} x^{2}$. This charge has to reach the surface by conduction through the jet cross section under the action of the electric field in the liquid, which requires a time $t_{c h} \sim \mathcal{C} / I_{b}$. Two possibilities appear.

(i) If the axial field in the liquid is of order $E_{\infty}$ before the surface charge reaches equilibrium and screens the liquid, then the dimensionless conduction current is $I_{b} \sim \Lambda E_{\infty} r_{s}^{2}$ and the time required to charge the surface is $t_{c h} \sim t_{e}\left(x / r_{s}\right)^{2} / \epsilon$, where $t_{e}=\epsilon / \Lambda$ is the electric relaxation time $\epsilon_{0} \epsilon / K$ nondimensionalized with the viscous capillary time $\mu a / \gamma$. In these conditions the normal electric field at the liquid side of the surface is $E_{n}^{l} \sim E_{\infty} r_{s} / x$, from the divergence-free condition $\boldsymbol{\nabla} \cdot \boldsymbol{E}^{l}=0$ with $E_{x}^{l} \sim E_{\infty}$, and the ratio of electric displacements at the two sides of the surface is $\epsilon E_{n}^{l} / E_{n} \sim \epsilon\left(r_{s} / x\right)^{2}$. This ratio is small, and $t_{c h}$ is large compared to $t_{e}$, if $x / r_{s} \gg \epsilon^{1 / 2}$. A small $\epsilon E_{n}^{l} / E_{n}$ means that $E_{n}$ is due mostly to the free charge accumulated at the surface; see Eq. (5a). The three terms of Eq. (5a) are of the same order, $E_{\infty} \epsilon r_{s} / x$, only in a first stage of the charging process, for times of order $t_{e}$, but the axial field induced by the surface charge is then small compared to $E_{\infty}$ and cannot screen the liquid.

(ii) These estimates predict a large $\epsilon E_{n}^{l} / E_{n}$ when $x / r_{s}$ $\ll \epsilon^{1 / 2}$, which is meaningless in view of Eq. (5a). In this case, corresponding to a very polar liquid, the polarization caused by the applied field $E_{\infty}$ keeps this field out of the liquid making $E_{n} \sim E_{\infty} x / r_{s}$ even before the accumulation of surface charge becomes noticeable in Eq. (5a). The normal field at the liquid side of the surface is $E_{n}^{l} \sim E_{n} / \epsilon$ before the surface charge reaches equilibrium; the density of conduction current normal to the surface is $\Lambda E_{n}^{l}$; and the characteristic time to attain equilibrium is $t_{c h}=t_{e}$, from the balance $\sigma / t_{e} \sim \Lambda E_{n}^{l}$ in the transport equation for the surface charge (5c) with $\sigma$ $\sim E_{n}$. The divergence-free condition $\boldsymbol{\nabla} \cdot \boldsymbol{E}^{l}=0$ gives now $E_{x}^{l}$ $\sim E_{n}^{l} x / r_{s} \sim E_{\infty}\left(r_{s} / x\right)^{2} / \epsilon \ll E_{\infty}$ during the charging, as was advanced before, and the conduction current is $I_{b} \sim \Lambda E_{x}^{l} r_{s}^{2}$ $\sim \mathcal{C} / t_{e}$.

In summary, the time it takes to charge the surface to equilibrium is $t_{c h} \sim t_{e} \max \left[\left(x / r_{s}\right)^{2} / \epsilon, 1\right]$.

The motion of the liquid has been disregarded in the preceding estimations. If a flow rate $Q$ runs through the jet, the characteristic velocity of the liquid is $v \sim Q / r_{s}^{2}$ and the residence time of a material particle in the stretch of the jet considered is $t_{r} \sim x r_{s}^{2} / Q$, which is the time available for the surface charge to approach its equilibrium. The condition $t_{r}$ $\sim t_{c h}$ imposes a relation between $r_{s}$ and $x$ that defines the current transfer region of the jet where electric conduction is effective at charging the surface to values of $\sigma$ of the order of its equilibrium value. The axial field induced by the free surface charge in the current transfer region is of the order of the applied field and partially balances it. The surface convection current in the current transfer region is $I_{s} \sim \sigma r_{s} v$ $\sim \mathcal{C} / t_{r}$, which is of the order of the conduction current. The conditions $I_{s} \sim I_{b}$ and $t_{r} \sim t_{c h}$ are equivalent definitions of the current transfer region.

A second relation between $r_{s}$ and $x$ comes from the balance of axial forces in the jet. For a stationary quasiunidirectional jet in the absence of inertia, this balance reads $[45,51]$

$$
\frac{\partial}{\partial x}\left(3 \pi r_{s}^{2} \frac{\partial v}{\partial x}\right)+\pi r_{s}^{2} \frac{\partial}{\partial x}\left(\tau_{n}^{e}-\frac{1}{r_{s}}\right)+2 \pi r_{s} \tau_{t}^{e}=0,
$$

where the three terms are the effective axial viscous force, the force due to the axial gradient of the pressure variation induced by the normal electric stress and the surface tension, and the force due to the electric shear stress. Here $\tau_{n}^{e}$ and $\tau_{t}^{e}$ are the components of the electric stress normal and tangent to the surface, given by the right-hand sides of Eqs. (4b) and (4c), respectively. In the region of interest the first term is of order $Q / x^{2}$ (using $v \sim Q / r_{s}^{2}$ ); the contribution of the normal electric stress to the second term is of order $E_{n}^{2} r_{s}^{2} / x \sim E_{\infty}^{2} x$ while the contribution of the surface tension, of $O\left(r_{s} / x\right)$, is expected to be much smaller; and the third term is of order $r_{s} \sigma E_{t} \sim E_{t} E_{\infty} x$. The second and third terms are of the same order when $E_{t} \sim E_{\infty}$, which happens for $\epsilon\left(r_{s} / x\right)^{2}$ small or of order unity, and the balance of these two forces and the viscous force requires $Q / x^{2} \sim E_{\infty}^{2} x$, hence $x \sim x_{0}=Q^{1 / 3} / E_{\infty}^{2 / 3}$. The third term of Eq. (9) is small compared to the second term when $\epsilon\left(r_{s} / x\right)^{2} \gg 1$ because then $E_{t} \ll E_{\infty}$ (cf. the estimate of $E_{x}^{l}$ for this case above). The balance of the first two terms of Eq. (9) gives the same result as before but, in the absence of a noticeable electric shear, a $\partial \tau_{n}^{e} / \partial x>0$ is required for the gradient of the electrically induced depression to push the liquid downstream. The numerical results show that this condition is realized only in a leading region of the jet, before $\tau_{n}^{e}$ attains its maximum and begins to decrease.

The electric shear is zero for a liquid of infinite conductivity, whose surface is an equipotential. Computations show that in this case the electric field at the surface [dotted curve of Fig. 5(b)] and the normal electric stress $\left(\tau_{n}^{e}=\frac{1}{2} E_{n}^{2}\right)$ increase monotonically along the jet, and that the radius of the jet decreases rapidly with streamwise distance. The latter result can be understood using the refined expression $\left(E_{n} r_{s} / x\right) \ln x / r_{s} \sim E_{\infty}$ (from slender body theory [49,50], used before without the logarithmic factor) to estimate the electric depression in the second term of Eq. (9). The balance of the first two terms of this equation gives $\ln x_{0} / r_{s} \sim\left(x / x_{0}\right)^{3 / 2}$.

For liquids of finite conductivity, the fast decrease of $r_{s}$ ends when the electric shear comes into play. This happens in the current transfer region where $t_{r} \sim t_{c h}$ if $\epsilon\left(r_{s} / x\right)^{2}$ is small or of order unity in this region, or where $\epsilon\left(r_{s} / x\right)^{2}=O(1)$ with $t_{r} \ll t_{c h}$ otherwise.

Using the estimates of $t_{c h}$ worked out before and leaving out logarithmic factors again, the condition $t_{r} \sim t_{c h}$ gives $x$ $=O\left(x_{0}\right)$ and $r_{s}=O\left(r_{s_{T}}\right)$ in the current transfer region, where

$$
\begin{gathered}
r_{s_{T}}=\frac{Q^{1 / 3}}{\Lambda^{1 / 4} E_{\infty}^{1 / 6}}, \quad I \sim \Lambda^{1 / 2} E_{\infty}^{2 / 3} Q^{2 / 3} \quad \text { if } \frac{\Lambda^{1 / 2}}{E_{\infty}} \gg \epsilon, \\
r_{s_{T}}=\frac{\epsilon^{1 / 2} E_{\infty}^{1 / 3} Q^{1 / 3}}{\Lambda^{1 / 2}}, \quad I \sim \frac{\Lambda Q^{2 / 3}}{\epsilon E_{\infty}^{1 / 3}} \quad \text { if } \epsilon \gg \frac{\Lambda^{1 / 2}}{E_{\infty}} \gg \epsilon^{1 / 2} .
\end{gathered}
$$

The two ranges of $\Lambda^{1 / 2} / E_{\infty}$ correspond to small and large values of $\epsilon\left(r_{s_{T}} / x_{0}\right)^{2}$, and the lower bound of $\Lambda^{1 / 2} / E_{\infty}$ in Eq. (10b) expresses the condition $x_{0} / r_{s_{T}} \gg 1$. The results for the 
electric current come from straightforward estimations of the surface convection and bulk conduction contributions, $I_{S}$ and $I_{b}$, which are of the same order in the current transfer region. The results for case (10a) coincide with estimates worked out elsewhere for a continuously fed jet [45]. Case (10b) is rather marginal and might not be fully realized owing to the effective lack of electric shear in the current transfer region. If the estimate $Q \sim E_{\infty}^{2}$ of Sec. III C 1 is carried into Eqs. (10), these results reduce to $x_{0} \sim 1$, and $\left(r_{s_{T}}, I\right)$ $\sim\left(E_{\infty}^{1 / 2} / \Lambda^{1 / 4}, \Lambda^{1 / 2} E_{\infty}^{2}\right)$ for $\Lambda^{1 / 2} / E_{\infty} \gg \epsilon$ and $\left(r_{s_{T}}, I\right)$ $\sim\left(\epsilon^{1 / 2} E_{\infty} / \Lambda^{1 / 2}, \Lambda E_{\infty} / \epsilon\right)$ for $\epsilon \gg \Lambda^{1 / 2} / E_{\infty} \gg \epsilon^{1 / 2}$.

Figure 9(b) shows that the electric currents for different values of $\Lambda$ and $\epsilon$ collapse reasonably well in a range of $E_{\infty}$ when they are scaled with the factor $\Lambda^{1 / 2} Q^{2 / 3}$ appearing in Eq. (10a). The scaled currents increase nearly as $E_{\infty}^{2 / 3}$, in agreement with Eq. (10a). When $E_{\infty}$ increases, the results for large values of $\epsilon$, for which the condition $\Lambda^{1 / 2} / E_{\infty} \gg \epsilon$ first breaks down, are the first to fall below the $2 / 3$ power law, though the prediction (10b) of a scaled current that decreases with $E_{\infty}$ is never realized. Figure 6(b) shows a similar collapse of the numerical data for the radius of the jet at the position of maximum $E_{n}$ when they are scaled with $Q^{1 / 3} / \Lambda^{1 / 4}$. Compare Fig. 6(b) with the unscaled radii in Fig. 6(a).

\section{Far jet}

Convection of the surface charge makes the largest contribution to the electric current of the jet downstream of the current transfer region. Conservation of the current then requires $\sigma v r_{s} \sim I$, and conservation of mass requires $v r^{2} \sim Q$. The applied field $E_{\infty}$ enters the jet when $\epsilon\left(r_{s} / x\right)^{2}$ ceases to be large downstream of the current transfer region. The balance of the first and third term of Eq. (9) then requires $r_{s}^{2} v / x^{2}$ $\sim r_{s} \sigma E_{\infty}$. These three conditions taken together determine the evolution of the radius, the velocity, and the density of surface charge of the jet with streamwise distance:

$$
r_{s}(x) \sim \frac{Q}{I^{1 / 2} E_{\infty}^{1 / 2} x}, \quad v(x) \sim \frac{I E_{\infty} x^{2}}{Q}, \quad \sigma(x) \sim \frac{I^{1 / 2}}{E_{\infty}^{1 / 2} x} .
$$

The electric stress normal to the surface can be estimated using these results and $E_{n} \sim \sigma$. It is $\tau_{n}^{e} \sim \max \left(\sigma^{2}, \epsilon E_{\infty}^{2}\right)$, where $\epsilon E_{\infty}^{2} / \sigma^{2} \sim \epsilon E_{\infty}^{3} x^{2} / I$, so that the contribution of the tangent field dominates the electric stress for $x \gg I^{1 / 2} \epsilon^{-1 / 2} E_{\infty}^{-3 / 2}$. The effect of the surface tension comes into play, making capillary breakup possible, when $1 / r_{s} \sim \tau_{n}^{e}$, which happens for $x$ $\sim \max \left(Q^{1 / 3} I^{1 / 6} / E_{\infty}^{1 / 2}, \epsilon E_{\infty}^{3 / 2} Q / I^{1 / 2}\right)$, where the radius of the jet is of order $r_{s_{c}}=\min \left(Q^{2 / 3} / I^{2 / 3}, 1 / \epsilon E_{\infty}^{2}\right)$. This gives rough estimates of the average volume of the droplets emitted by the jet $\left(r_{s_{c}}^{3}\right)$ and the average emission frequency $\left(Q / r_{s_{c}}^{3}\right)$, up to numerical factors. Unfortunately these estimates cannot be assessed by comparison with the numerical results of Sec.
III A. The computations cannot be reliably extended to the high values of $\Lambda$ that would be necessary for such comparison because their precision degrades when the jet becomes very thin.

The effect of the inertia of the liquid has been left out of Eqs. (2)-(7) on the assumption that the Reynolds number $\operatorname{Re}=\rho \gamma a / \mu^{2}$ is small. However, the inertia will play a role in a long jet even if it is negligible in the attached drop and in a leading region of the jet. The inertia of the liquid would lead to a term $\operatorname{Re} r_{s}^{2} \partial v^{2} / \partial x$ on the right-hand side of Eq. (9) (see Ref. [51]). The ratio of this term to the first term of Eq. (9) can be estimated as $\operatorname{Re} I E_{\infty} x^{3} / Q$ using Eq. (11). Therefore the balance of viscous and electric shear forces used to derive Eq. (11) should be modified if the length of the jet is not small compared to $\left(Q / \operatorname{Re} I E_{\infty}\right)^{1 / 3}$.

\section{CONCLUSIONS}

The elongation of a drop attached to a metallic plate following the application of a uniform electric field has been described numerically for liquids of finite electrical conductivity in the absence of inertial effects. The elongation of the drop leads to a hydrostatic equilibrium if the applied field is smaller than a certain critical value, or to the emission of daughter droplets at higher fields. In the latter case, and in the ranges of parameters that have been investigated, the computation reproduces an evolution often observed in experiments. The drop develops a conical shape around its tip and ejects a fine jet that emits small charged droplets. The flow rate and the electric current carried by the stream of droplets are determined by the flow and the transport of electric charge in the attached drop and in a leading region of the jet, where the solution is nearly time independent and amenable to a simplified analysis. The effect of the finite conductivity of the liquid comes into play in a region at the beginning of the jet where the solution departs from that for a liquid of infinite conductivity. In this region, the electric field normal to the surface reaches a maximum, the rate of variation of the radius of the jet with streamwise distance decreases substantially, and convection of the surface charge becomes the mechanism responsible for most of the electric current. The electric stresses dominate surface tension stresses in this region and in the attached drop when the applied field is moderately larger than its critical value and the electrical conductivity is high. The balance of electric and viscous stresses gives then a flow rate that increases as the square of the applied field, while the order of the electric current can be determined from a qualitative analysis of the current transfer region of the jet.

\section{ACKNOWLEDGMENTS}

This work was supported by the Spanish Ministerio de Educación y Ciencia through Project No. DPI2007-66659C03-02. 
[1] C. T. R. Wilson, Philos. Trans. R. Soc. London, Ser. A 221, 194 (1921)

[2] C. T. R. Wilson and G. I. Taylor, Proc. Cambridge Philos. Soc. 22, 728 (1925).

[3] A. G. Bailey, Electrostatics Spraying of Liquids (Wiley, New York, 1988).

[4] J. Fernández de la Mora, Annu. Rev. Fluid Mech. 39, 217 (2007).

[5] J. B. Fenn, M. Mann, C. K. Meng, S. K. Wong, and C. Whitehouse, Science 246, 64 (1989).

[6] Lord Rayleigh, Philos. Mag. 14, 184 (1882).

[7] K.-Y. Li, H. Tu, and A. K. Ray, Langmuir 21, 3786 (2005).

[8] J. Fernández de la Mora, J. Colloid Interface Sci. 178, 209 (1996).

[9] M. A. Abbas and J. Latham, J. Fluid Mech. 30, 663 (1967).

[10] D. G. Roth and A. Kelly, IEEE Trans. Ind. Appl. IA-19, 771 (1983).

[11] D. C. Taflin, T. L. Ward, and E. J. Davis, Langmuir 5, 376 (1989).

[12] X. Feng, M. J. Bogan, and G. R. Agnes, Anal. Chem. 73, 4499 (2001).

[13] C. B. Richardson, A. L. Pigg, and R. L. Hightower, Proc. R. Soc. London, Ser. A 422, 319 (1989).

[14] D. B. Hager and N. J. Dovichi, Anal. Chem. 66, 1593 (1994).

[15] D. B. Hager, N. J. Dovichi, J. Klassen, and P. Kebarle, Anal. Chem. 66, 3944 (1994).

[16] D. Duft, T. Achtzehn, R. Müller, B. A. Huber, and T. Leisner, Nature (London) 421, 128 (2003).

[17] T. Achtzehn, R. Müller, D. Duft, B. A. Huber, and T. Leisner, Eur. Phys. J. D 34, 311 (2005).

[18] M. Cloupeau and B. Prunet-Foch, J. Electrost. 22, 135 (1989).

[19] M. Cloupeau and B. Prunet-Foch, J. Aerosol Sci. 25, 1021 (1994).

[20] J. Fernández de la Mora and I. G. Loscertales, J. Fluid Mech. 260, 155 (1994).

[21] S. I. Betelú, M. A. Fontelos, U. Kindelán, and O. Vantzos, Phys. Fluids 18, 051706 (2006).

[22] G. I. Taylor, Proc. R. Soc. London, Ser. A 280, 383 (1964).

[23] C. T. O'Konski and H. C. Thacher, Proc. R. Soc. London, Ser. A 133, 565 (1931).

[24] S. S. Abbi and R. Chandra, Proc. Natl. Inst. Sci. India, Part A 22, 363 (1956).
[25] P. R. Brazier-Smith, Phys. Fluids 14, 1 (1971).

[26] M. J. Miksis, Phys. Fluids 24, 1967 (1981).

[27] O. A. Basaran and L. Scriven, Phys. Fluids A 1, 799 (1989).

[28] N. Dubash and J. Mestel, Phys. Fluids 19, 072101 (2007).

[29] R. L. Grimm and J. L. Beauchamp, J. Phys. Chem. B 109, 8244 (2005).

[30] L. Oddershede and S. R. Nagel, Phys. Rev. Lett. 85, 1234 (2000).

[31] S. N. Reznik, A. L. Yarin, A. Theron, and E. Zussman, J. Fluid Mech. 516, 349 (2004).

[32] R. T. Collins, J. J. Jeremy, M. T. Harris, and O. A. Basaran, Nat. Phys. 4, 149 (2008).

[33] J. R. Melcher and G. I. Taylor, Annu. Rev. Fluid Mech. 1, 111 (1969).

[34] D. A. Saville, Annu. Rev. Fluid Mech. 29, 27 (1997).

[35] L. D. Landau and E. M. Lifshitz, Electrodynamics of Continuous Media (Pergamon, Oxford, 1960).

[36] G. K. Batchelor, An Introduction to Fluid Dynamics (Cambridge University Press, Cambridge, 1967), p. 132.

[37] X. D. Shi, M. P. Brenner, and S. R. Nagel, Science 265, 219 (1994).

[38] F. J. Higuera, Phys. Fluids 19, 072113 (2007).

[39] R. Juraschek and F. W. Röllgen, Int. J. Mass. Spectrom. 177, 1 (1998).

[40] P. Nemes, I. Marginean, and A. Vertes, Anal. Chem. 79, 3105 (2007).

[41] M. S. Wilm and M. Mann, Int. J. Mass Spectrom. Ion Process. 136, 167 (1994)

[42] M. S. Wilm and M. Mann, Anal. Chem. 68, 1 (1996).

[43] M. S. Alexander, M. D. Paine, and J. P. W. Stark, Anal. Chem. 78, 2658 (2006).

[44] M. D. Paine, M. S. Alexander, and J. P. W. Stark, J. Colloid Interface Sci. 305, 111 (2007).

[45] F. J. Higuera, J. Fluid Mech. 558, 143 (2006).

[46] F. J. Higuera, Phys. Fluids 19, 012102 (2007).

[47] F. J. Higuera, Phys. Rev. E 77, 026308 (2008).

[48] F. J. Higuera, J. Fluid Mech. 484, 303 (2003).

[49] H. Ashley and M. Landahl, Aerodynamics of Wings and Bodies (Addison-Wesley, Reading, MA, 1965), Chap. 6.

[50] E. J. Hinch, Perturbation Methods (Cambridge University Press, Cambridge, England, 1991).

[51] J. J. Feng, Phys. Fluids 14, 3912 (2002). 